\title{
Investigation of Harmonic Issues in Distribution Grids due to High Share of Electric Vehicles
}

\author{
Diego Di Domenico, Artjoms Obushevs, Petr Korba \\ Institute of Energy Systems and Fluid Engineering \\ Zurich University of Applied Science \\ Winterthur, Switzerland \\ obus@zhaw.ch,korb@zhaw.ch
}

\begin{abstract}
-with the advent of power electronics, the application of solid-state electronics for the control and conversion of electric power is substantially increasing at the expenses of simple rotating machines and linear loads. This leads to power quality problems due to the sinewave distortion of current and voltage caused by such power-electronics-based equipment. Consequently, the investigation and understanding of harmonic emissions caused by those devices become of major importance in order to assure a continuous and reliable operation of the electrical distribution grid. The following paper aims to investigate the harmonic issues in distribution grids, due to power electronic devices with particular focus on electric vehicle chargers.
\end{abstract}

Keywords- distribution system impacts, electric vehicles, harmonic emission, power system harmonics

\section{INTRODUCTION}

Power-electronic-based devices are becoming more and more present in the electric grid. Those devices are used for the conversion and control of electric power and have made available new technologies for both power generation and consumption. In particular, power electronics has allowed for new methods of electricity power production by means of renewable sources as well as the advent of new types of loads. Nevertheless, some downsides arise and, among others, the increase of voltage and current distortion generated by the nonlinearity of these devices which may constitute a problem when excessively high. Some examples of new harmonic sources which can be found in the distribution grid and whose penetration can be expected to increase are: compact fluorescent lamps (CFLs) [1], light-emitting diode (LED) lamps, distributed photovoltaic (PV) systems [2], adjustablespeed-drive-based home appliances and electric vehicle (EV) chargers [3],[4].

Despite a wide range of devices can produce high order frequencies in the grid, because of the availability of data, this work sees its main focus on EVs chargers. EVs with a significant on-board energy storage which can be recharged via the distribution network are commonly battery electric vehicles (BEVs) and plug-in hybrid vehicles (PHEVs). Due to the similarity of the battery charging process [5], in this paper such vehicles are all referred to as electric vehicles (EVs). EVs are usually equipped with batteries having an energy storage capacity up to several tens of $\mathrm{kWh}$. In order to recharge the batteries in a reasonable time-frame, high recharging powers can be reached up to a few hundreds of $\mathrm{kW}$ for ultra-fast charging [5]. Since the batteries operate in DC, to restore the energy in an EV from the electric grid, a power conversion $\mathrm{AC}$ to DC is needed. This is done by power-electronic rectifiers located either inside the car or in a dedicated EV charging station. These rectifiers can cause current harmonic distortions and thus negatively affect the grid. Consequently, the ongoing adoption of EVs, along with other power electronics devices, has raised questions over their impact on the distribution network. The electric grid is thus facing news challenges, and the investigation and understand of the effects of powerelectronic-based devices is of increasingly importance in order to ensure a continuous and reliable power distribution. In this regard, the aim of this paper is to investigate the additional losses deriving from a distortion of the pure fundamental sinewave for which the grid is meant for. This, with particular emphasis on harmonic emissions of EV chargers at the distribution grid level

The carried-out investigations are based on two datasets provided by the supply and disposal company of the city of Winterthur, Stadtwerk Winterthur (SW) [6]. Those data have been investigated in order to understand the harmonic emission of EV chargers. The analyses of harmonic losses have been carried out by means of the DIgSILENT analysis software PowerFactory (PF).

The following paper is structured as follows. In the second chapter Approach and Methodology is presented. In the first part of this chapter the measurements data are visualized and a first evaluation on the harmonic emission of the EV chargers is discussed. In the second part, the adopted approach to evaluate the harmonic losses is presented. The third chapter presents and visualizes the results of data investigation. In this section are shown the results drawn by the data investigation along with the findings obtained by the harmonic losses simulations in PF. In the final chapter conclusion with a recapitulation of the conducted work is given.

\section{APPROACH AND METHODOLOGY}

SW has provided two datasets. The first dataset contains various charging information such as power, voltage, current and harmonics content during charging time for multiples types of cars. The second data set provide similar information but for multiples charging stations over a much longer period of time, from November 2018 to April 2019. These two datasets have 
been then filtered and organized in MATLAB in order to extract the harmonics content for both voltage and current. It follows an investigation on the harmonics content variation as a function of the EVs charging rate, where some interesting behaviors have been identified with regards to the current harmonics. Based on the available information and the peculiarities observed, some current spectrums at specific charging power rates have been selected to conduct further studies in PF. To do so, for each harmonic spectrum of interest, the data of current magnitude and phase have been extracted and adjusted in order to be implemented in PF.

Lastly, the different spectrums have been implemented in PF as current loads, allowing to gain a better understanding on the share of losses due to the harmonic emission and if this could arise potential issues. For this, a simple LV electric grid has been modelled.

\section{A. Harmonic analysis of different EVs models}

These first datasets of measurements were taken using a power quality analyzer Fluke MEMOBOX 808. The EV charger employed is manufactured by $\mathrm{ABB}$ and allows $\mathrm{DC}$ charging up to $50 \mathrm{~kW}$. Measurement values are provided each 30 seconds and are performed on the following EVs: BMW i3 (4 measurements); Hyundai IONIQ; Kia Soul (2 measurements); Mercedes B250e; Mitsubishi i-MiEV; Nissan Leaf (2 measurements); Renault Zoe (2 measurements); Volkswagen e-Golf; Volkswagen e-up.

\section{1) Evaluation of current harmonics}

In order to have an overall overview of the harmonic emission Figure 1 shows the current THD levels for all vehicle models during the charging period. It is noticeable a similar behavior for all models, with an average THDi level of $6.35 \%$. The harmonic distortion level remains approximately constant with the increase of the current (and thus the charging power) with a moderate dispersion for low current values. This is particularly the case for the Mercedes B250. Another vehicle to slightly differ from the overall trend is the Renault Zoe. One of the measurements for this EV shows a significant lower THDi level.

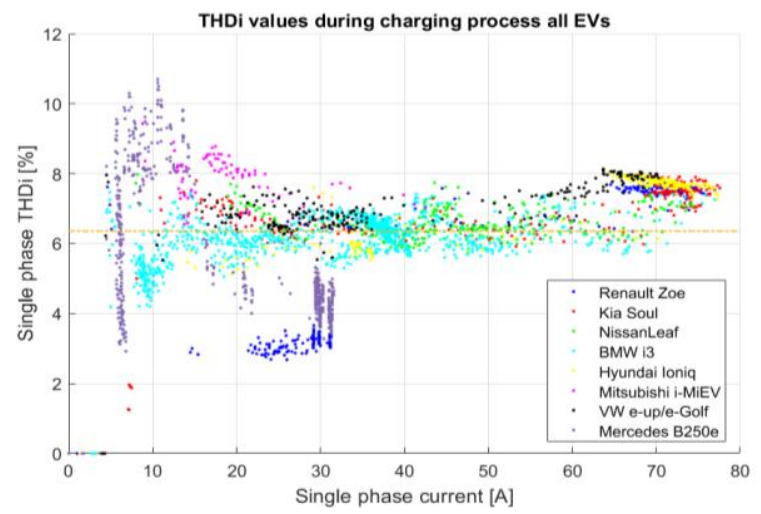

Fig. 1. THDi levels during charging time for all 3 phases, all vehicles together

For both the Mercedes B250e and one of the measurements of the Renault Zoe, it is noticeable a current limit at about 32A. Indeed, $32 \mathrm{~A}$ corresponds to the maximum current for a power charging level type 2 as defined in the standard IEC 618511 [7]. A charging power of level 2 allows a maximum power of $22 \mathrm{~kW}$. Looking at Figure 2 where all charging profiles are displayed, one can clearly see that these two vehicles were limited to a charging power of $22 \mathrm{~kW}$. The next power limit defined in the standard IEC $61851-1$ is up to $120 \mathrm{~kW}$ for level 3. Level 3 and 4 differentiate from the others since power transfer management may takes place in the charging station and not in the vehicle. This may suggest that the two EVs with different THDi behavior have been charged via the AC/DC converter equipped in the car. The remaining majority of EVs may have been charged via the same AC/DC converter of the charging station. The harmonic behaviors of the onboard rectifiers may differ from the one of the charging station, this would explain why the majority of the measured THDi levels are similar. Moreover, according to [8], the Mercedes B250e does not allows to be charged via an external DC station but solely with a charging level of type 2 , thus further validating the above assumption.

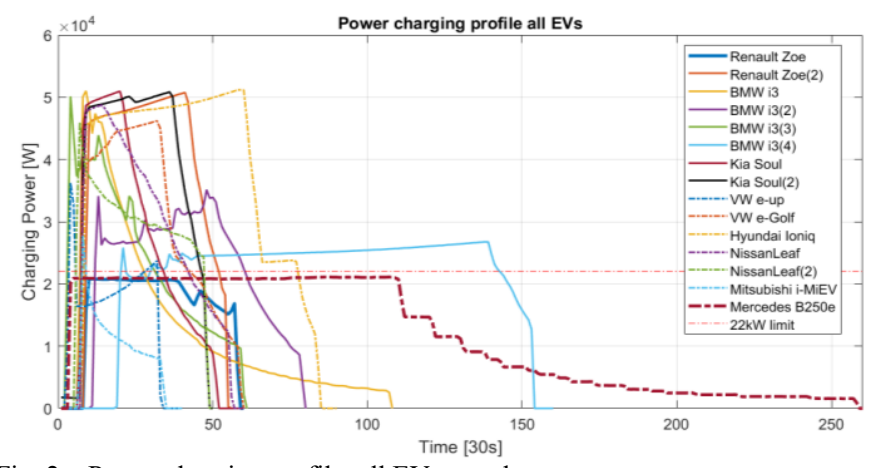

Fig. 2. Power charging profile, all EVs together

An additional analysis can be carried out on the current spectrum during the charging period. In this regard, Figure 3 shows the average current harmonic distribution over the whole charging period for all EVs. Note that the fundamental component has been scaled down by a factor of 10 for better visualization. It is noticeable that even harmonics are almost completely absent, indicating half-wave symmetry of the current waveform. Triplen harmonics are also of little concern because of their negligible magnitude. This spectrum composition coincides with a 6-pulse bridge rectifier. In fact, as mentioned in the IEEE Std 519-2014 [9], three-phase rectifierbased converter produces dominant harmonic currents at orders of $\mathrm{p}(\mathrm{n} \pm 1)$, where $\mathrm{n}$ is a simple counter, $\mathrm{n}=1,2,3$ etc., and $\mathrm{p}$ is the pulse-order of the rectifier ( $p=2,6,12,18,24$, etc.).

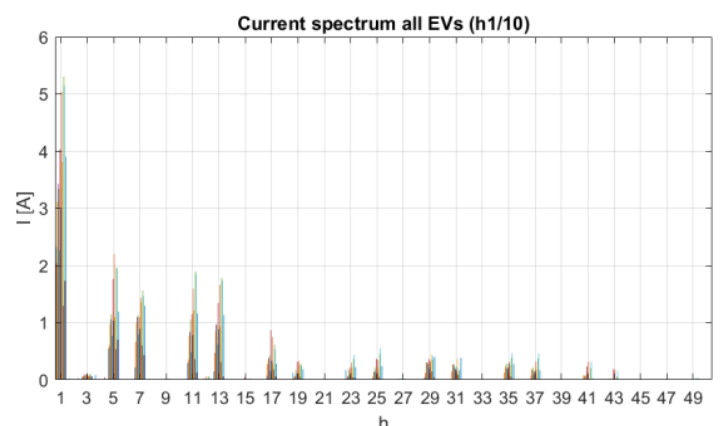

Fig. 3. Average current spectrum during charging process averaged over the three phases, all EVs together 


\section{2) Evaluation of voltage harmonics}

Figure 4 shows the average voltage harmonic distribution over the whole charging period for all EVs. The displayed values are averaged over the three phases and expressed as percentage of the nominal voltage, i.e. $230 \mathrm{~V}$. Note that the fundamental component is reduced by a factor of 10 for better representation. The average voltage THD values are of $1.5 \%$ and no higher THDu values of $2.17 \%$ have been recorded. This is fully compliant with the maximum recommended value by IEEE Std 519-2014 [9] and DACHCZ [10] of 8\% for LV installations.

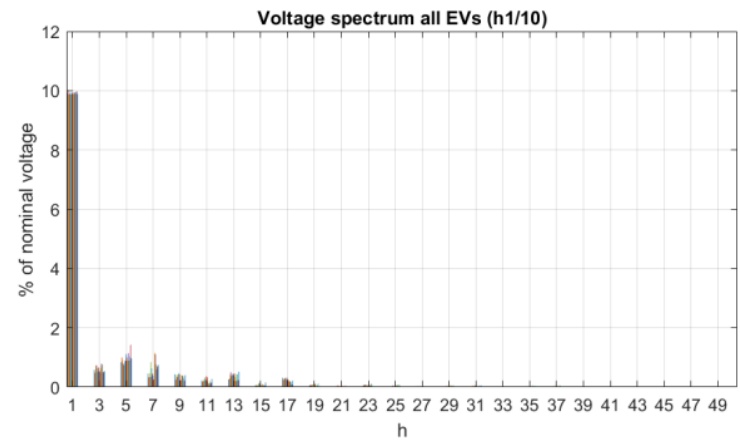

Fig. 4. Average voltage harmonic components during charging process averaged over the three phases, all EVs together

Considering each single harmonic component and comparing it with the recommended compatibility levels by DACHCZ specified in [10], one can see that the voltage harmonic contents is fully compliant with the standard.

\section{B. Harmonic analysis of $S W$ charging station}

These measurements were taken at the PCC using the power quality analyzer PQ-Box 200, where about $30 \mathrm{EV}$ chargers are installed and exploited by SW [6]. For this reason, this data set only considers the charging behavior of the vehicles employed by the enterprise, namely the Renault Kangoo Z.E. and Renault Zoe. EV charging behavior is presented in Figure 5. Unlike the charger employed for the measurement of multiples EVs, those chargers do not allow DC charging. The period under consideration starts on 5.11.2018 and ends on 9.4.2019. Because of the large amount of data, values with a time interval of $10 \mathrm{mins}$ are used.

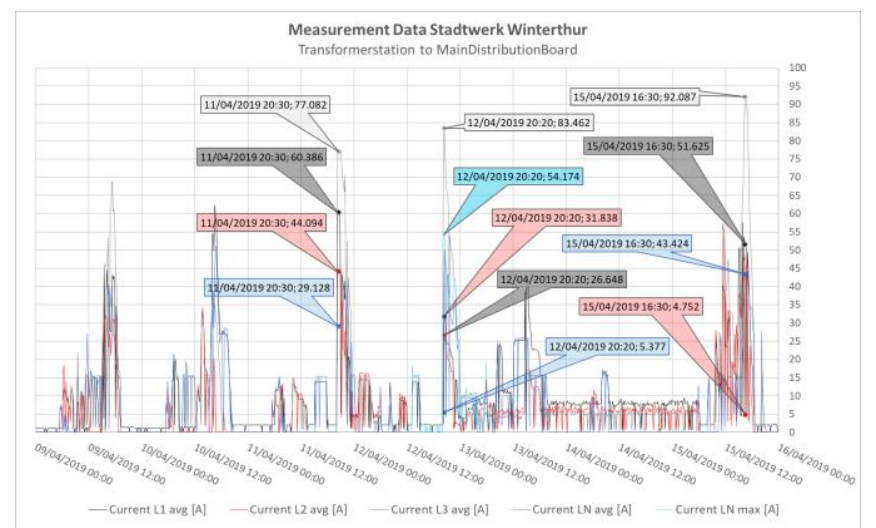

Fig. 5. EVs load measurement data in ampere from SW Simplified Grid, at Main Distribution Board (9. April to 16. April)

\section{1) Evaluation of current harmonics}

As made for the different EVs, also for this dataset the current THD levels have been plotted as a function of the current, as showed in Figure 6. This has been done for each phase and considering only the measurements recorded during the charging times, i.e. at least a minimum current value of $0.65 \mathrm{~A}$ was drawn from the grid. One can immediately notice the interesting distribution of THDi values. For all three phases THDi is subjected to an exponential-like increase as the RMS current decrease toward zero. We can therefore deduce that for low power consumption the current waveform is highly distorted. On one hand, this could be of minor concern since the current magnitude is not high enough to negatively affect the grid. On the other hand, due to the high frequencies present, even a relatively low magnitude may negatively affect the nearby equipment and generate losses. Nevertheless, for a phase current over 10-15A, the THDi level comes back to a more typical value. Indeed, the average THDi level for the three phases for a phase current above $10 \mathrm{~A}$ is $6.8 \%$.

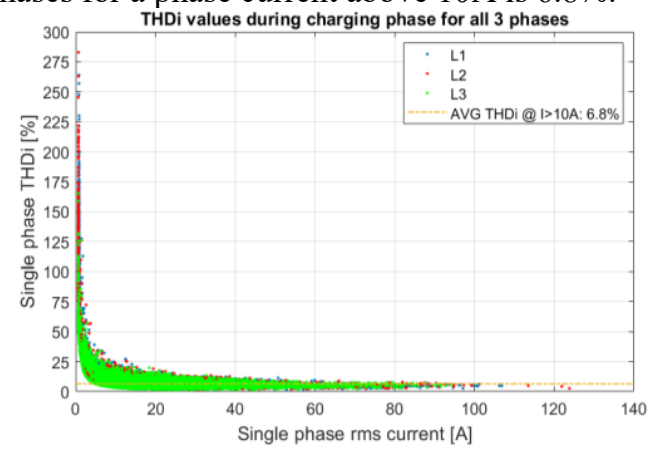

Fig. 6. THDi levels during charging time for all 3 phases

Figure 7 shows the average current spectrum for the three phases, again, note that the fundamental amplitude is scaled down by a factor of 10 . In contrast to the first dataset, the harmonic components are typical of a single-phase 2-pulse bridge rectifier, i.e. $2(\mathrm{n} \pm 1)$. Another interesting aspect is that the average current for the three phases is unequal. This is mainly due to the fact, that those chargers do not allow DC charging, as it was the case for the measurements with multiples EVs. Consequently, if a car support single-phase charging only, the car onboard converter may be connected directly to one of the three phases, thus creating a load unbalance and explaining the harmonic characteristic of a single-phase rectifier. In the case of a charging pole providing DC charging, the latter is most probably connected to the three phases and the power is consequently better distributed.

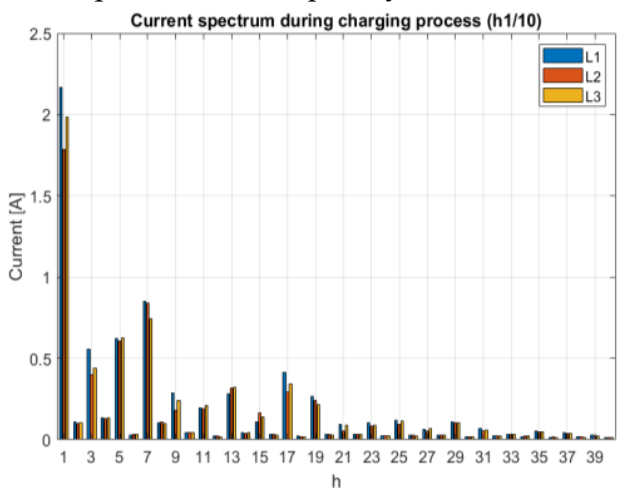

Fig. 7. Average current spectrum during charging time 
The unbalances between the three phases can also be observed by analyzing the neutral conductor in Figure 8 . Indeed, a significant high frequency of occurrence for current values of about $16 \mathrm{~A}$ is shown. In fact, $16 \mathrm{~A}$ corresponds to the maximum current for single-phase power charging level of type 1 as defined in the standard IEC 61851-1 [7]. Suggesting that the employed vehicles are often charged by a single phase. Furthermore, an additional step at about 32A can be noticed. This suggests that the vehicles are not equally distributed over the three phases and asymmetries of two (and more) EVs per phase are present.

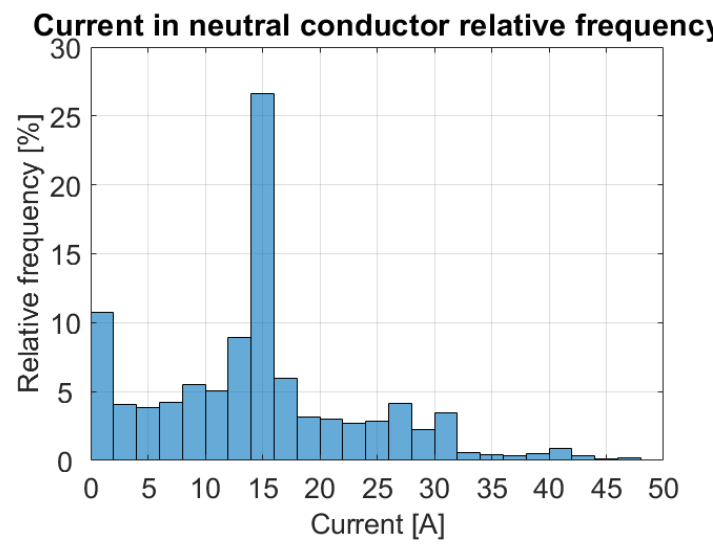

Fig. 8. Relative frequency of current in neutral conductor during charging time

\section{2) Evaluation of voltage harmonics}

Concerning the voltage distortion, Figure 9 shows its average spectrum during the charging process. Also, for this dataset, the spectrum has a low distortion, with average THD values for all the three phases of about $1.6 \%$. No particular trends have been observed and all the harmonic components are fully compliant with the DACHCZ recommendations.

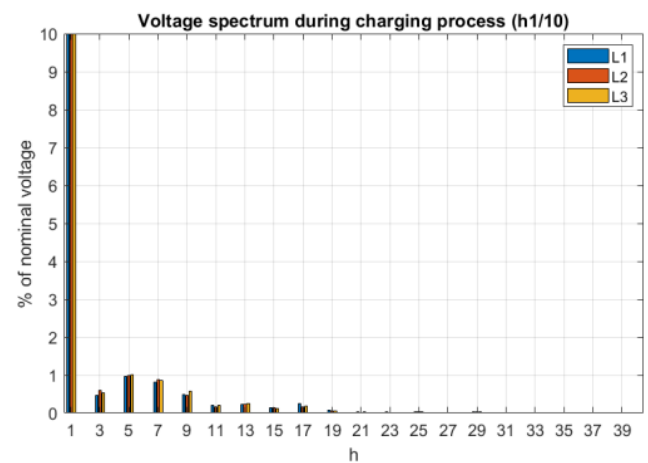

Fig. 9. Average voltage spectrum during charging time

\section{Power Factory simulations}

In order to evaluate the share of losses due to the current distortion, different current spectrums have been extracted and implemented in PF in order to perform multiple harmonic load flow calculations. This calculation consists of a load flow computation for all frequencies for which harmonic sources are defined. This allows to consider the impedance variation of the grid elements at different frequencies, i.e. each harmonic component is subject to the impedances of the grid elements corresponding to its specific frequency. The impedance behavior of elements such as cables, lines and transformers can be configured to vary accordingly with the frequency. In this investigation, a simple grid composed by a cable connecting the current harmonic source to an external grid was considered. The current harmonic source is used to emulate the EV charging stations' behavior.

On account of the obtained simulation results with real data, it has been decided to carry out a second series of simulation in PF but with a fundamental current constant and balanced for the three phases. This is because, the real data have shown low THDi values for significant current amplitude, making it difficult to evaluate the harmonic share of losses. Instead, the second series of simulations better shows how harmonic losses vary for different current distortion. To carry out this simulation, real spectrums from data have been selected with various THDi levels, while the fundamental current has been kept unchanged to 20A for all simulation series.

\section{1) Network model}

The network model is designed to evaluate the losses caused by the current distortion. In this regard, a LV cable has been used to connect the external grid to the EVs chargers, as illustrated in Figure 10. In a practical case, this will represent the losses in the cable used to connect the EVs chargers to the PCC.

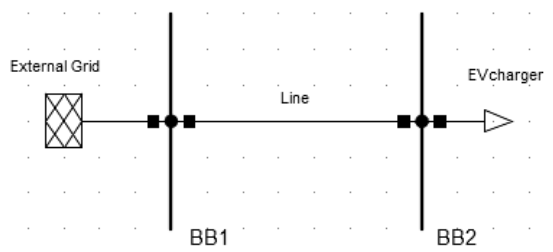

Fig. 10. Power Factory network model

The selection of the cable has been made based on its rated voltage and current according to the available models provided in the PF library. Since the exact maximum load of the EV chargers is not known, the selection of the cable has been made based on the data. The highest recorded current per phase was approximately $120 \mathrm{~A}$, considering a security factor of 1.5 accounting for a possible expansion of the vehicle fleet, this gave a rated current of about $180 \mathrm{~A}$. The NYBY $3 \times 50 / 25 \mathrm{~mm} 2$ $0.6 / 1 \mathrm{kV}$ power cable fulfill this requirement, with an underground rated current of 185A [11].

In order to account for the frequency-dependent parameters, in $\mathrm{PF}$ is possible to associate to the resistance and inductance of the line a frequency characteristic. Two types of characteristic may be used, a frequency polynomial characteristic or a userdefined frequency table. In our case, since no specific information were available, a frequency polynomial characteristics provided in PF has been used. The most similar cable available in the library which has a defined frequency characteristic is the $20 \mathrm{kV}$ NEKBA $3 \times 1 \times 70$ power cable. Consequently, in the model, the $20 \mathrm{kV}$ NEKBA cable characteristic has been used to model the frequency dependency of both resistance and inductance. The relative resistance increases as a function of the harmonic rang is shown in Figure 11 and is given by the equation: 


$$
R(f) / R_{D C}=1+a \cdot\left(\frac{f}{f_{\text {nom }}}-1\right)^{b}
$$

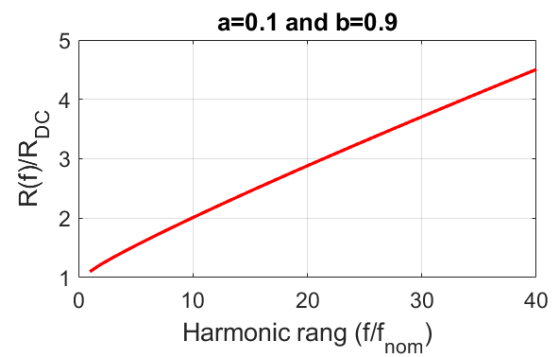

Fig. 11. Resistance frequency characteristic for $20 \mathrm{kV}$ NEKBA $3 \times 1 \times 70$ cable

\section{2) Definition of harmonic spectrums}

The EV chargers are simulated by a current source load with their corresponding spectrum. In order to input the data in $\mathrm{PF}$, the harmonic amplitudes were expressed in percentage of the fundamental and the phase angles were referred to the phase angle of the 1st harmonic.

The conversion of the amplitudes is quite straightforward, while the change of reference angle was considered more carefully since the simple operation of subtracting $\phi 1$ to each $\phi \mathrm{h}$ is wrong. The correct operation corresponds to change the time origin, in such a way that the fundamental has an angle equal to zero. This transformation leads to the harmonic angle $\phi$ h' which is the proper phase to implement in PF and is calculated as follows:

$$
\phi_{h}{ }^{\prime}=\phi_{h}-h \cdot \phi_{1}
$$

Where, $\phi_{h}$ is the harmonic phase angle; $\phi_{1}$ is the fundamental component phase angle; $h$ is the harmonic rang.

Note that the available measurements may use a different reference to express the current angles, e.g. referenced to the voltage, in such case a transformation may be needed.

\section{3) Considered spectrum}

The current harmonic spectrums implemented in PF have been retrieved from the second dataset, i.e. the SW. For the real case simulation, 11 spectrums have been selected based on their THDi and power level. The selected points are shown in Figure 12.

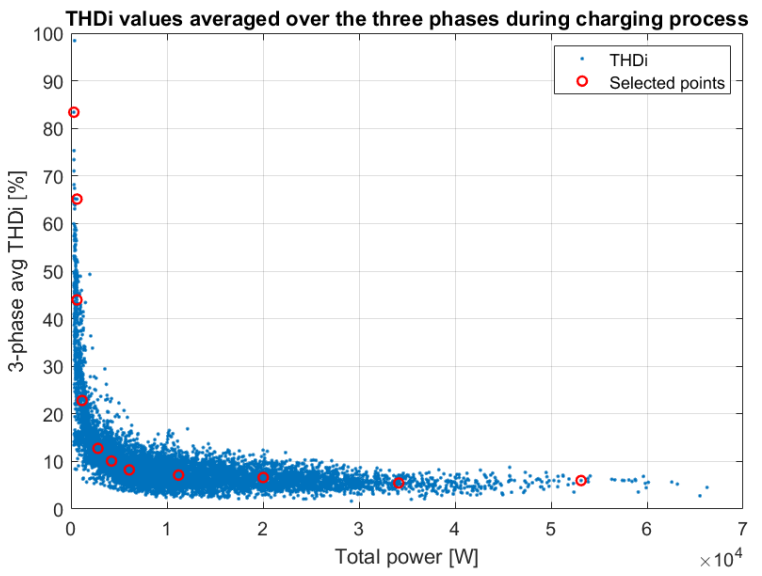

Fig. 12. Selected points corresponding to the spectrums implemented in PF
In Table 1 is presented a summary of these measurements. It is noticeable that the current is not always identical over the three phases, the same applies for the THDi which is usually higher for low current levels.

\begin{tabular}{|c|c|c|c|c|c|c|c|c|}
\hline Point & $\begin{array}{l}\text { Ptot } \\
{[W]}\end{array}$ & $\mid \begin{array}{c}\text { Qtot } \\
\text { [Var] }\end{array}$ & IL1 [A] & IL2 [A] & IL3 [A] & |THDi L1 [\%] & THDi L2 [\%] & THDi L3 [\%] \\
\hline 1 & 300 & 731 & 0,86 & 0,71 & 1,03 & 99,4 & 93,1 & 42,3 \\
\hline 2 & 535 & 1'322 & 0,84 & 0,71 & 2,45 & 98,9 & 91,7 & 25,6 \\
\hline 3 & 623 & 1'495 & 0,84 & 0,71 & 2,80 & 99,0 & 90,0 & 24,4 \\
\hline 4 & 1'157 & 3'221 & 0,81 & 6,26 & 0,55 & 93,1 & 11,5 & 74,4 \\
\hline 5 & $2^{\prime} 804$ & $5^{\prime} 664$ & 0,94 & 0,70 & 11,34 & 73,5 & 86,1 & 5,38 \\
\hline 6 & $4^{\prime} 225$ & 9'415 & 0,80 & 18,56 & 0,74 & 90,0 & 6,37 & 53,15 \\
\hline 7 & 6'072 & $7^{\prime} 852$ & 17,09 & 0,71 & 9,54 & 5,55 & 89,2 & 6,29 \\
\hline 8 & $11^{\prime} 201$ & 9'285 & 8,51 & 22,90 & 22,06 & 14,26 & 5,76 & 5,53 \\
\hline 9 & $20^{\prime} 019$ & $15^{\prime} 875$ & 42,17 & 18,76 & 36,66 & 5,03 & 9,69 & 6,13 \\
\hline 10 & $34^{\prime} 128$ & $11^{\prime} 813$ & 50,23 & 52,92 & 53,78 & 4,94 & 5,376 & 5,596 \\
\hline 11 & $53^{\prime} 122$ & $20^{\prime} 921$ & 91,48 & 77,76 & 77,55 & 5,17 & 6,18 & 5,93 \\
\hline
\end{tabular}

TABLE I. SPECIFICATION OF THE SELECTED POINTS

An example of spectrum definition in PF for the 5th chosen point is given in Figure 13.

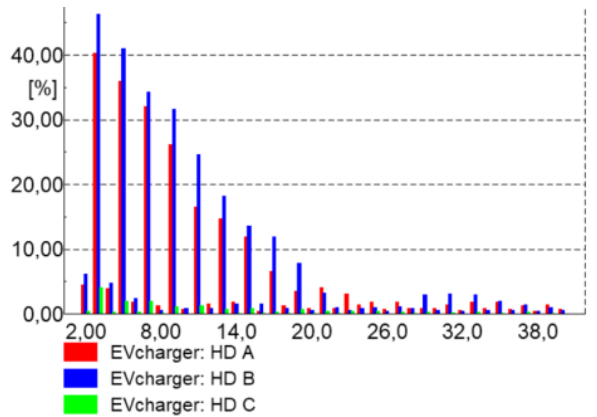

Fig. 13. Harmonics distribution per phase for the 5 th selected point

Regarding the second series of simulations with identical fundamental current, the spectrums have been chosen and defined similarly as before but with focus on taking a wide selection of THDi levels, thus allowing to run multiples simulations with various levels of distortion. Note that for this case the same spectrums have been implemented over the three phases since the interest is to evaluate the losses caused by a current distortion and not to replicate the real behavior.

\section{RESULTS OF DATA INVESTIGATION}

Hereafter are presented the results derived from the data investigation and the harmonic losses evaluation conducted in PF. Some comparison and analogy with the literature are equally presented.

\section{A. Dataset 1: Multiple EVS}

An investigation on the effect of power consumption on the voltage distortion has shown that no particular increase on the voltage harmonics is caused by the distorted current. This can be visualized in Figure 14, where two voltage harmonics distributions have been plotted for the first dataset with multiples vehicles. One spectrum shows the average harmonic components during the charging process while the second one shows the average magnitudes when no power is consumed from the grid. It becomes clear that the voltage distortion remains almost unchanged with an increase of the power demand, suggesting that the voltage harmonics are most probably coming from the external grid and no significant changes are attributable to the charging station. 


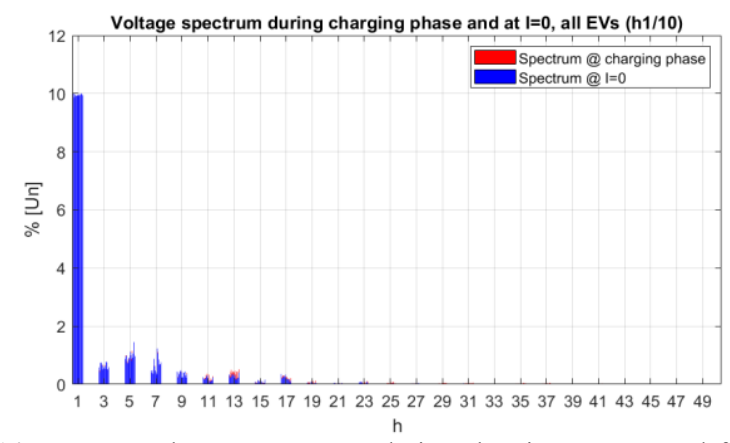

Fig. 14. Average voltage components during charging process and for $\mathrm{I}=0 \mathrm{~A}$ averaged over the three phases, all EVs together

This can also be seen by looking at the correlation between THDu and power has presented in Figure 15. Indeed, there is no substantial variation of the voltage distortion with the increase of the power consumption.

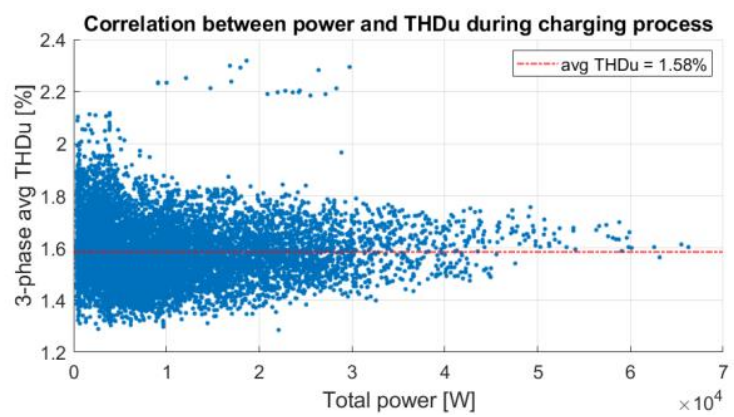

Fig. 15. Correlation between power and voltage THD during charging process

\section{B. Dataset 2: Long time measurement}

Consequently, to the high THDi levels recorded, it has been decided to quantify the time duration at which the grid is subjected to such current distortion. Even though the current magnitude is relatively low for high THDi values, and thus not constituting a major concern in terms of losses, the same may not be the case for electronic equipment or metering devices. The concluded time at which the system is subject to high distortion, defined as THDi $>15 \%$, is about $4.5 \%$ of the time. This is the average value for the three phases when exclusively considering the time instants during the charging process, i.e. for a phase current higher than $0.65 \mathrm{~A}$. The time instants under consideration can be visualized in Figure 16. If one considers the whole period of measurement this value drastically increases to a phase average of $66.9 \%$. This is because whenever a car is not connected a highly distorted base current of about $0.5 \mathrm{~A}$ to $0.6 \mathrm{~A}$ is continuously taken from the grid, presumably due to the own consumption of the charging stations. If the metering device does not properly measure the current and due to the relatively long time at which the current has a significant level of distortion, the metering error could become relevant even if the current magnitude is low. To better quantify the possible consequence, let us consider a metering error causing an underestimation of the consumed power of $20 \%$ over a year. For a phase current of $0.6 \mathrm{~A}$, the standby consumption $\mathrm{P}_{\text {stby }}$ of the EV chargers is equal to $414 \mathrm{~W}$. Consequently, the uncounted energy consumed by the EV chargers will be:

$E_{\text {error }}=P_{\text {stby }} \cdot 20 \% \cdot 24 \mathrm{~h} \cdot 365$ days $\cdot 66.9 \%=414 \mathrm{~W} \cdot 0.2 \cdot 8760 \mathrm{~h} \cdot 0.669=485 \mathrm{kWh}$
In term of costs, considering an energy price of $20 \mathrm{cts} / \mathrm{kWh}$, this would translate in a monetary loss by the energy provider of $97 \mathrm{CHF} /$ year.

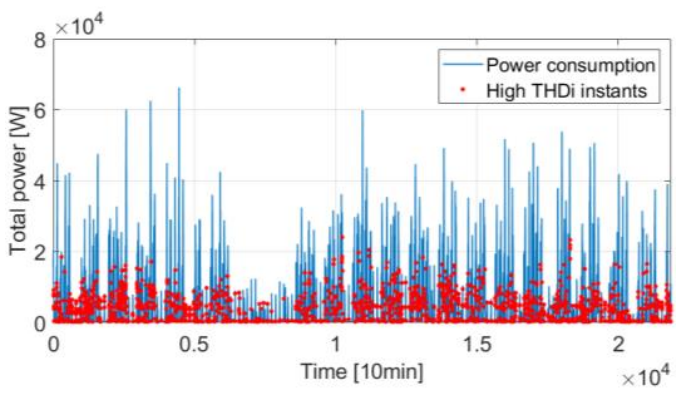

Fig. 16. Time instants at THDi $>15 \%$ during chargin process

\section{Comparison of $E V s$ data with literature}

Despite the significant difference of the THDi behavior for the two datasets, similar patterns can be found in the literature, showing that this is not an isolated case but rather it depends on the type of EV charging method. Indeed, Kattman et al. in [12] have recorded similar characteristics. Where vehicle A has a similar THDi behavior as the one measured with the long-time dataset, while vehicles $\mathrm{B}$ and $\mathrm{C}$ show a more constant current distortion as it was the case with the multiple EVs dataset. Zhang et al. in [13] have also recorded a significant difference in current distortion depending on the current magnitude, measuring THDi values up to $130 \%$ overnight, when the EV chargers are rarely used.

It is also interesting to observe the similarity that can be found with other power electronic devices. In this regard, some PV inverters exhibit a similar THDi behavior as the one observed with the long-term dataset, this, although the power flow of an inverter is the opposite of an EV charger rectifier. Papers [14] and [15] illustrate the above-mentioned similarities.

\section{Results of Power Factory simulations}

\section{1) Real case simulation}

The real case simulations have shown that the harmonics at low power have a major influence on the losses and may constitute up to $65 \%$ of the total losses. Nevertheless, once the charging power increase and the current distortion decrease, their share becomes lower than $1 \%$ and thus negligible compared to the losses at fundamental frequency. This can be seen in Figure 17 where the harmonic losses share is plotted against the total consumed power.

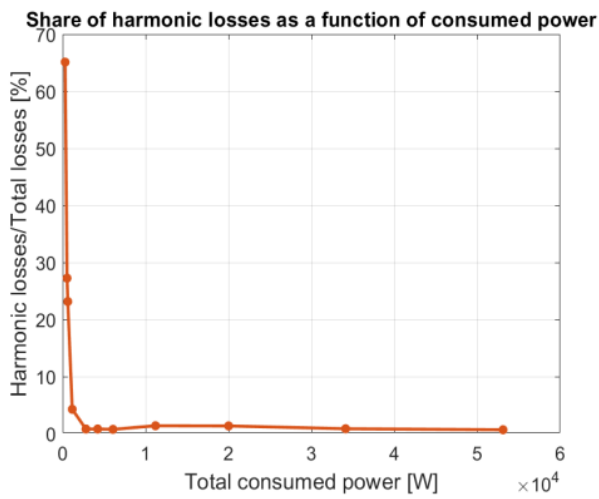

Fig. 17. Harmonic losses share as a function of the consumed power by the EV chargers 
The distortion impact on the losses can also be seen in Figure 18, where it becomes clear that with high THDi the harmonic losses become substantial and need therefore to be considered in the dimension of the equipment. Nonetheless, it is important to point out that for the considered case such THDi levels occur solely at low currents and thus the losses are negligible.

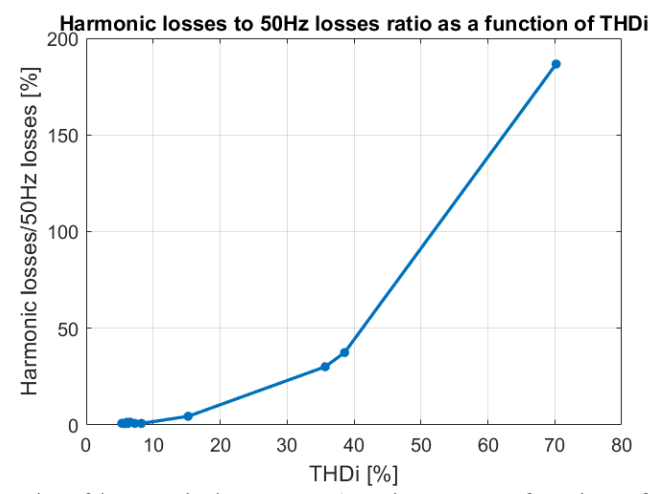

Fig. 18. Ratio of harmonic losses to $50 \mathrm{~Hz}$ losses as a function of the current THD, real case scenario

Until now, the harmonic losses have been expressed relatives to the remaining losses, showing a higher loss share for high current distortion. Nevertheless, in these measurements, the actual harmonic losses are higher for low THDi values since it is during those instants the current magnitude is at its maximum. This means that even if the harmonics components are lower relative to the fundamental, the increase in magnitude is such to generate higher losses. In those cases, where the current has an important fluctuation, expressing the distortion by means of the THD can be misleading. In fact, it would be more convenient to use the TDD, which will better quantify the severity of the harmonic distortion in terms of losses. To better visualize this effect, in Figure 19 the average THDi and TDDi trends are superimposed to the harmonic losses as a function of the power consumed by the EV chargers. It can be seen how the TDD properly follows the harmonic losses behavior while the THD progress in the opposite direction.

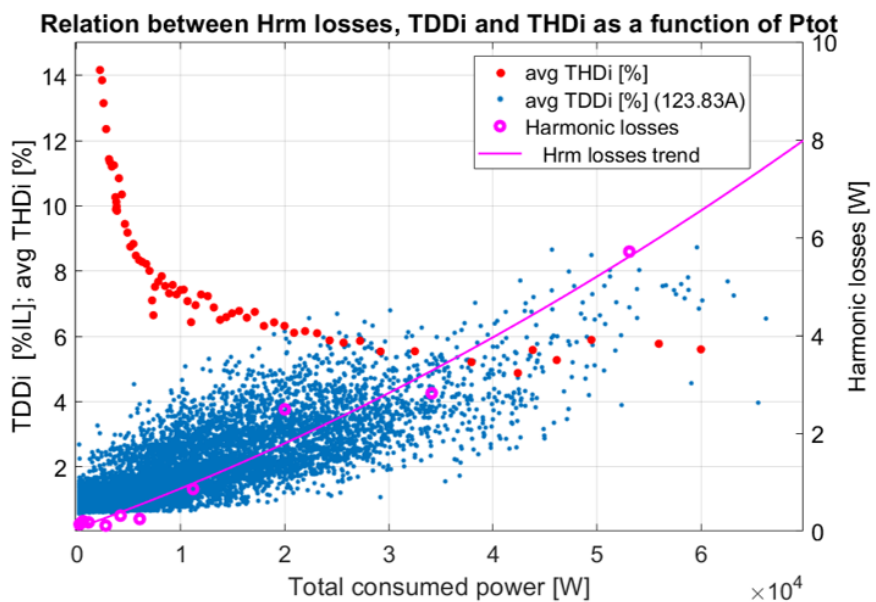

Fig. 19. Relation between harmonic losses, TDDi and THDi as a function of the consumed power
2) Balanced system simulation with varying current distortion

Maintaining a constant current for different levels of current distortion has allowed to better understand the effect of harmonics, avoiding the condition of low current for high THDi values as presented in the real case simulations. According to Figure 20, for the selected frequency characteristic of the power cable, it is possible to conclude that the losses increase quadratically in the range of distortion investigated.

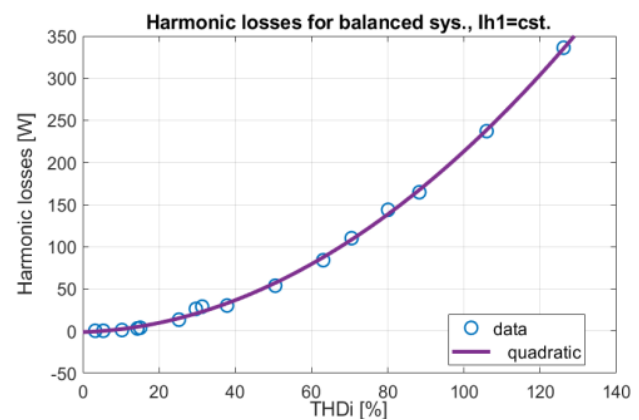

Fig. 20. Harmonic losses as a function of the current distortion with constant fundamental current

This result is further confirmed by a study conducted by Y. G. Sahin et al. in [16], where an analogous behavior was achieved. In this investigation multiples effects such as skin effect, proximity effect and the cable temperature were considered.

In terms of harmonic losses share, the results have shown an s-shaped increase along with the growth of the current distortion as illustrated in Figure 21. Consequently, it can be deduced a relatively low contribution to the overall losses up to approximately $15 \% \mathrm{THDi}$, followed by an important increase. It this therefore recommendable to remain under a THDi level of $15 \%$.

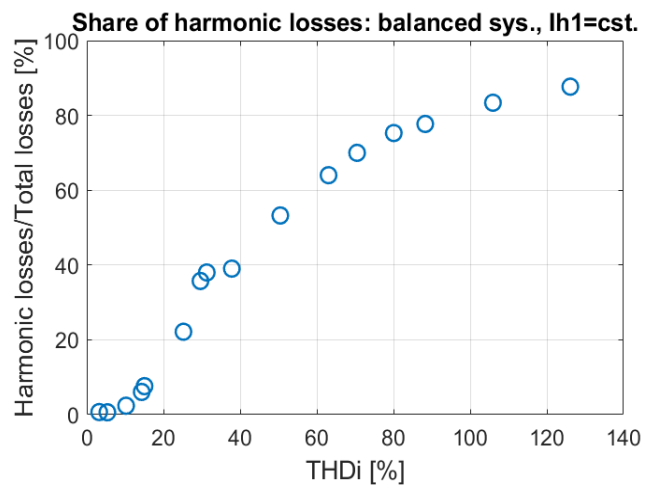

Fig. 21. Share of harmonic losses as a function of the current THD for a constant fundamental current value

\section{CONCLUSION}

This work has its main focus on the investigation of harmonic emission of EVs chargers. Two data sets from SW of EVs charging profiles have been investigated. The first data set contains various charging information for different EVs manufacturers. The second data set provides similar information for a single EVs manufactures but over a longer period of time. These data have been analyzed in order to gain a better understanding of the harmonic emission produced by 
EVs chargers. The harmonic spectrum for both voltage and current is investigated for different car models and various charging power. Finally, the losses generated by the additional harmonic emissions have been investigated for a simple network. The goal being to gain a better understanding of the share of losses due to the harmonic penetration and if this could arise potential issues. The simulations have been carried out using the DIgSILENT analysis software PowerFactory.

The data investigation has shown that voltage distortion is not of major concern and no substantial variations caused by the current have been detected. Concerning the current, different THD values depending on the charging method employed have been observed, this has been found to be consistent with the literature. According to the EV charging method high THD values close to $100 \%$ were reported for low charging power. Nevertheless, the simulations have revealed that this is most likely not an issue in terms of losses due to the low current magnitude. Despite that, this could be problematic for sine wave-sensitive electronics and metering devices. A second investigation on the extent of harmonic losses has shown that for THD values higher than 15\%-20\% the losses due to the current distortion considerably increase, potentially damaging other equipment in the grid. Furthermore, it has been concluded that the TDD better quantify the severity of the harmonic distortion in term of losses. This, as opposed to the THD, which can be misleading in the case of high distorted current with negligible magnitude.

\section{ACKNOWLEDGMENT}

This research is part of the activities of the Swiss Centre for Competence in Energy Research on the Future Swiss Electrical Infrastructure (SCCER-FURIES), which is financially supported by the Swiss Innovation Agency (Innosuisse SCCER program)

\section{REFERENCES}

[1] H. Dghim, A. El-Naggar and I. Erlich, "Harmonic distortion in low voltage grid with grid-connected photovoltaic," 2018 18th International Conference on Harmonics and Quality of Power (ICHQP), Ljubljana, 2018, pp. 1-6, doi: 10.1109/ICHQP.2018.8378851.

[2] Y. Li, W. Wang, B. Yuwen, S. Wei, W. Su and Z. Lu, "Harmonic Model and Propagation in Power System: A Review," IECON 2019 - 45th Annual Conference of the IEEE Industrial Electronics Society, Lisbon, Portugal, 2019, pp. 6147-6151, doi: 10.1109/IECON.2019.8927241.

[3] H. Sharma, M. Rylander and D. Dorr, "Grid Impacts Due to Increased Penetration of Newer Harmonic Sources," in IEEE Transactions on Industry Applications, vol. 52, no. 1, pp. 99-104, Jan.-Feb. 2016, doi: 10.1109/TIA.2015.2464175.

[4] A. Bosak, A. Bosak, L. Kulakovskyi and T. Oboronov, "Impact of EV Chargers on Total Harmonic Distortion in the Distribution System Network," 2019 IEEE 6th International Conference on Energy Smart Systems (ESS), Kyiv, Ukraine, 2019, pp. 329-333, doi: 10.1109/ESS.2019.8764244.

[5] L. Kütt; E. Saarijärvi, M. Lehtonen, H. Mõlder, J. Niitsoo, "Current harmonics of EV chargers and effects of diversity to charging load current distortions in distribution networks," 2013 International Conference on Connected Vehicles and Expo (ICCVE), pp. 726-731, 2013.

[6] Stadtwerk Winterthur, "Main Page, Stadtwerk Winterthur," 2019. Available: https://stadtwerk.winterthur.ch/

[7] S. Habib, M.M. Khan, F. Abbas, et al "A Comprehensive Study of Implemented International Standards, Technical Challenges, Impacts and Prospects for Electric Vehicles," IEEE Access, vol. 6, pp. 1386613890, 2018.
[8] "Electric vehicle database," [Online]. Available: https://evdatabase.org/car/1013/Mercedes-B-250e. [Accessed 1001 2020].

[9] IEEE Recommended Practice and Requirements for Harmonic Control in Electric Power Systems," IEEE Std 519-2014 (Revision of IEEE Std 519-1992), p. p10, 2014.

[10] G. Bartak, H. Holenstein, and J. Meyer, Eds., "D-A-CH-CZ Technical Rules for the Assessment of Network Disturbances", 2nd Edition. VEÖ, VSE, CSRES, VDN, 2007.

[11] Eland Cables, "NYBY Cable," 2020. [Online]. Available: https://www.elandcables.com/info/search?searchtext=NYBY. [Accessed 25 January 2020].

[12] C. Kattmann, K. Rudion, and S. Tenbohlen, "Detailed power quality measurement of electric vehicle charging infrastructure," CIRED - Open Access Proceedings Journal, vol. 2017, no. 1, pp. 581-584, 2017.

[13] Y. Zhang, D. Yu, G. Zhang, H. Wang and J. Zhuang, "Harmonic Analysis of EV Charging Station Based on Measured Data," 2020 IEEE/IAS Industrial and Commercial Power System Asia (I\&CPS Asia), Weihai, China, 2020, pp. 475-480, doi: 10.1109/ICPSAsia48933.2020.9208498.

[14] F. Batrinu, G. Chicco, J. Schlabbach, F. Spertino, "Impacts of gridconnected photovoltaic plant operation on the harmonic distortion," In: Proceedings IEEE Melecon 2006, Benalmádena, Málaga, Spain, 16-19 May 2006, pp. 861-864.

[15] G. Chicco, R. Napoli and F. Spertino, "Experimental Evaluation of the Performance of Grid-Connected Photovoltaic Systems," Proceedings of the 12th IEEE Mediterranean Electrotechnical Conference, vol. 3, pp. 1011-1016, 2004.

[16] Y. G. Sahin and F. Aras, "Investigation of Harmonic Effects on Underground Power Cables," International Conference on Power Engineering, Energy and Electrical Drives, pp. 589-594, 2007.

Diego Di Domenico received his BSc degree in electrical engineering with major in electric power from the School of Engineering and Architecture of Fribourg, Switzerland, in 2018. He carried out the Bachelor thesis at the Lawrence Berkeley National Laboratory, California, in the Accelerator Technology and Applied Physics Division (ATAP). Where he has modeled and simulated the electrical circuit of a compact radio-frequency particle accelerator. Since 2018, He is pursuing an MSc degree in electrical engineering at the Institute of Energy Systems and Fluid Engineering of the Zurich University of Applied Science ZHAW in Winterthur, Switzerland.

Dr. Artjoms Obushevs received the BSc, MSc, $\mathrm{PhD}$ degree in electrical engineering from the Riga Technical University, in 2008, 2010 and 2014 respectively. Since 2018, He is a Research Associate in the Electric Power Systems and Smart Grids group at the Institute of Energy Systems and Fluid Engineering of the Zurich University of Applied Science ZHAW in Winterthur, Switzerland. His main research is focused on methods of mathematical modelling of electrical networks and systems elements; development of power systems planning; dynamic optimization methods and decision systems. Author and Co-Author of more than 50 peer-reviewed journal and conference papers in the field of power systems.

Prof. Dr. Petr Korba received the Dipl.-Ing. degree in electrical engineering from Czech Technical University, Prague, Czech Republic, in 1995, and the Dr.-Ing. degree from the University of Duisburg, Germany, in 1999. He was a Member of Academic Staff with the Institute of Science and Technology, The University of Manchester, in 2001. He joined ABB Switzerland Ltd. He held different positions in the business unit power system automation. He worked as a Principal Scientist with ABB Corporate Research Ltd., for more than ten years. Since 2008, he has been a Lecturer with ETH Zurich. He was a Professor of electric power systems with the Zurich University of Applied Sciences, in 2012, where he is currently the Head of the Electric Power Systems Group and the Deputy Head of the Institute of Energy Systems and Fluid Engineering. He is also the Co-Director of the Swiss Competence Centre of Energy Research (SCCER, Grids and Components).

Has published over 100 articles in international journals and at international conferences in the field of automatic control and electric power systems. He also authored and co-authored over 100 US and European patents and patent applications and was nominated for the Best European Patent Award for achievements in the wide-area monitoring and control of electrical power systems in 2011. 